\title{
El valor intangible de la innovación de las marcas del distribuidor
}

\author{
Carmen ABRIL \\ Departamento de Comercialización e Investigación de Mercados \\ Universidad Complutense de Madrid \\ cabril@ccee.ucm.es \\ Joaquín SÁNCHEZ-HERRERA \\ Departamento de Comercialización e Investigación de Mercados \\ Universidad Complutense de Madrid \\ joaquin.sanchez@ccee.ucm.es \\ Diana GAVILÁN \\ Departamento de Comercialización e Investigación de Mercados \\ Universidad Complutense de Madrid \\ diana@soleste.es \\ Roberto MANZANO \\ Departamento de Comercialización e Investigación de Mercados \\ Universidad Complutense de Madrid \\ rmanzano@ccee.ucm.es \\ Maria AVELLO \\ Departamento de Comercialización e Investigación de Mercados \\ Universidad Complutense de Madrid \\ mavello@emp.ucm.es
}

Recibido: 12-09-2012

Aceptado: 02-12-2012

\section{RESUMEN}

Las marcas del distribuidor han experimentado un extraordinario crecimiento en todo el mundo, alcanzando en España una cuota de mercado cercana al 40\%. Estas marcas han evolucionado en el tiempo aumentando el surtido, desarrollando un portafolio más sofisticado, y en muchos casos adoptando estrategias de innovación y mejora de la calidad. Los objetivos de este estudio son: en primer lugar investigar la percepción que tienen los consumidores españoles sobre la innovación de las marcas del distribuidor y en segundo lugar, analizar cómo estas percepciones influyen en la 
creación de valores intangibles atribuidos a las marcas del distribuidor, a las enseñas que las respaldan y la prescripción de los consumidores.

Palabras clave: innovación, marcas del distribuidor, imagen de marca, boca-oído.

\title{
The intangible asset of private labels innovation
}

\begin{abstract}
Private labels have experience extraordinary growth worldwide, reaching in Spain a market share close to $40 \%$. Private labels have clearly evolved over time increasing the range, developing a more sophisticated portfolio and in many cases adopting innovation and quality improvement strategies. The objectives of this paper are: firstly to investigate consumer perceptions of private-label innovativeness and secondly to analyze how these perceptions influence the creation of intangible assets related to the perception of private labels, retailers and other consumers prescriptions.
\end{abstract}

Keywords: innovation, private labels, store image, word of mouth.

Sumario: 1. Introducción. 2. Las marcas del distribuidor y los beneficios de la innovación. 3. Hipótesis a investigar. 4. Investigación empírica y resultados. 5. Conclusiones e implicaciones para la gestión

\section{INTRODUCCIÓN}

¿Son las marcas del distribuidor agentes impulsores de la innovación? La formulación de esta pregunta produce inevitablemente una gran polémica en los círculos académicos y empresariales. A menudo se argumenta que las marcas del distribuidor, también denominadas marcas blancas, basan su estrategia en reproducir las novedades de las "marcas de fabricante"1, generando en muchos casos situaciones que podrían rozar la ilegalidad. Aunque es cierto que en sus orígenes las marcas del distribuidor utilizaron la imitación de las marcas líderes como estrategia competitiva en la actualidad nos enfrentamos a un panorama muy diferente.

Las marcas del distribuidor han experimentado una gran transformación volviéndole más sofisticadas y la percepción del consumidor sobre ellas ha cambiado radicalmente (Kumar y Steenkamp, 2007). Las causas de este cambio en la percepción de los consumidores se pueden atribuir a diversos factores, entre

\footnotetext{
${ }^{1}$ La denominación marca de fabricante se utiliza por contraposición a la de marca del distribuidor o marca blanca, denotando aquellas marcas que son comercializadas por empresas cuyo objetivo principal es la producción y comercialización de productos en el mercado.
} 
ellos a una mejora de su calidad en general, a una mayor amplitud en la oferta y surtido, pero también a la innovación. Algunas marcas del distribuidor como las de Mercadona en España, Tesco en Inglaterra o Loblaws en Estados Unidos deben gran parte de su éxito a la innovación como estrategia competitiva y diferenciadora del resto de marcas.

Aunque existen diversos estudios que acreditan el crecimiento en la innovación de las marcas del distribuidor (DelVecchio, 2001; Mintel, 2009), las investigaciones sobre sus efectos en el mercado son escasas.

Algunas recientes investigaciones abordan por primera vez el efecto de la innovación desde el punto de vista de del contexto competitivo que se crea entre las marcas de distribuidor y las marcas de fabricante (Geykens et al., 2010). Sin embargo, resulta llamativa la escasez de estudios que aborden el impacto de la innovación desde la perspectiva del consumidor. ¿Percibe el consumidor la innovación de las marcas del distribuidor? ¿Cómo influyen estas percepciones en la relación entre el consumidor, la marca del distribuidor y la enseña?

El objetivo de este estudio es doble. En primer lugar investigar la percepción que tienen los consumidores españoles sobre la innovación de las marcas del distribuidor y en segundo lugar, analizar cómo estas percepciones influyen en la creación de valores intangibles atribuidos a las marcas del distribuidor, a las enseñas que las respaldan y a la prescripción de los consumidores.

El trabajo se estructura en dos partes. En la primera se describe el entorno de las marcas del distribuidor en el mercado español y los beneficios esperados de la innovación. En la segunda parte se presenta un estudio empírico que contrasta las relaciones propuestas entre las variables descritas. El trabajo finaliza ofreciendo algunas recomendaciones de interés para distribuidores y fabricantes que se desprenden de los resultados de la investigación.

\section{LAS MARCAS DEL DISTRIBUIDOR Y LOS BENEFICIOS DE LA INNOVACIÓN}

La marca del distribuidor ha alcanzado en España un $40 \%$ del total de las ventas del mercado de gran consumo, lo que sitúa a este país como el segundo con más peso en marca de distribuidor de Europa (Symphony IRI, 2012).

La razón del crecimiento de esta cuota se atribuye parcialmente a los resultados alcanzados por las enseñas que más se apoyan en ellas, como es el caso de Mercadona, así como también al diferencial de precios ofertado respecto a la marca de fabricante, -cuyos precios son superiores en un 30\%- (Abril et al., 2010). Sin embargo, no son éstas las únicas razones del crecimiento experimentado por las marcas del distribuidor. Cabe destacar el aumento del surtido -alrededor de un $30 \%$ más de referencias que en $2007-$, así como su creciente actividad innovadora. 
Ambos hechos contribuyen a aumentar la ventaja competitiva respecto a las marcas de fabricante.

Paralelamente en España las marcas de fabricante redujeron su actividad innovadora en un $75 \%$, mientras que la marca del distribuidor la mantuvo, como se desprende del estudio SymphonyIRI (2012), en el que se define la actividad innovadora como el lanzamiento de nuevos productos al mercado que aportan una característica nueva, independiente del grado de innovación que posea, excluyendo las promociones.

Esta tendencia respecto al lanzamiento de nuevos productos se hace eco de lo que sucede en otros mercados, como Estados Unidos, donde las marcas del distribuidor fueron responsables en 2011 del 31,4\% de los nuevos productos lanzados en alimentación (Datamonitor, 2012), lo que representa el doble que en 2010 y más del triple que en 2009.

El fenómeno de la innovación de las marcas del distribuidor tiene gran relevancia por su impacto en la dinámica del mercado de gran consumo. Sin embargo las investigaciones que abordan cómo afecta la innovación a la percepción de los consumidores sobre las marcas del distribuidor y sus enseñas son todavía muy escasas.

Si se adopta el punto de vista del distribuidor, se podría esperar que algunos de los efectos de la innovación impulsada por las marcas de distribuidor fueran asimilables a los ya generados por medio de la incorporación al surtido de marcas Premium procedentes del distribuidor (Verhoef et al., 2004), puesto que en ambos casos se trata de aumentar el atractivo de la oferta. Los ejemplos sobre el impacto de la incorporación de estas marcas Premium al surtido son múltiples, tal y como aparecen recogidos en la literatura: brindan al distribuidor la posibilidad de incrementar sus márgenes, aumentando el surtido propio con productos exclusivos (Ailawadi y Keller, 2004; Garretson et al., 2002; Pauwels y Srinivasan, 2004; Sprott y Shimp, 2004).

Sin embargo, existe un amplio consenso en torno a la idea de que, en las empresas los beneficios esperados de la innovación suelen trascender el puro valor económico. De hecho, las compañías utilizan índices objetivos basados en indicadores que están relacionados con el consumidor y la introducción en el mercado además de la medición de los resultados financieros (Molina-Castillo y Munuera-Aleman 2009). Específicamente en el área de marketing se observa que a través de la innovación se pueden obtener otros beneficios intangibles como la mejora de la imagen de marca, el establecimiento de relaciones más estrechas con los consumidores, el refuerzo de la reputación de la marca o potenciar su liderazgo (Aaker , 1991; 1996). 


\section{HIPÓTESIS A INVESTIGAR}

En esta investigación se analizará en primer lugar el grado en que el consumidor español percibe como innovadoras las marcas del distribuidor, y posteriormente el impacto que dicha percepción tiene sobre los constructos: imagen de la enseña, confianza en la marca y tendencia a la prescripción de la misma.

La percepción de una marca como innovadora abarca diversos conceptos, tales como un surtido de productos único, novedoso y original; calidad comparable a las marcas líderes y una sensación de sorpresa y novedad en sus lanzamientos (Moreau et al., 2001; Olshavsky y Spreng, 1996).

En el caso particular de las marcas del distribuidor el efecto de la innovación revierte en primer lugar sobre sí misma, mejorándose la imagen de marca del distribuidor (Sriram et al., 2007). Ahora bien, dada la relación probada entre la imagen de marca del distribuidor y la imagen de la enseña que la respalda (Ailawadi y Keller, 2004), cabe esperar que los beneficios en términos de imagen que se desprenden de la percepción de innovación de la marca del distribuidor, también se transfieran a la imagen de la enseña (Collins-Dodd y Linley, 2003).

Esta transferencia de imagen se produce porque la imagen de una enseña está afectada por la calidad percibida de los productos que vende (Grewal et al., 1998). Las marcas del distribuidor se venden exclusivamente en los establecimientos propiedad de los detallistas a los que pertenecen. Por tanto, las marcas de distribuidor son percibidas como una extensión de la imagen de la enseña y por tanto su innovación puede considerarse una fuente de diferenciación para la enseña que las respalda.

Por tanto formulamos la hipótesis de que la percepción de las marcas del distribuidor como innovadoras afectará positivamente a la imagen de la enseña a la que pertenecen.

H1: Las marcas del distribuidor percibidas como innovadoras afectarán positivamente la imagen de su enseña.

La confianza es un constructo clave en el que se fundamenta la relación de los consumidores con los distribuidores o detallistas. La confianza en la marca es una de las herramientas más poderosas en los servicios, y en concreto en la distribución detallista (Berry, 2000). Cuanto mayor sea la satisfacción del consumidor, su compromiso y la confianza que le inspire la enseña, mayor será la fortaleza de su relación con el distribuidor (Morgan y Hunt, 1994, Johnson y Selnes, 2004).

Aaker (1996) señala que la innovación percibida forma parte de las asociaciones de la marca relacionadas con el liderazgo. En este sentido la percepción de la marca del distribuidor como innovadora ejercerá una influencia positiva en su percepción de liderazgo. Esta percepción liderazgo por parte del consumidor 
incrementará a su vez la confianza del consumidor hacia la marca del distribuidor. Por ello se formula la siguiente hipótesis:

H2: Las marcas del distribuidor percibidas como innovadoras generan más confianza en el consumidor.

Un aspecto relevante de la marca innovadora es su capacidad de suscitar sorpresa derivada de las novedades de sus lanzamientos (Moreau et al., 2001; Olshavsky y Spreng, 1996). La generación de sorpresa suele estar acompañada por la satisfacción que este hecho conlleva (Abril et al., 2009). La satisfacción se asocia con la habilidad de un proveedor para hacer que su cliente hable positivamente de él, llegando a prescribirle. Por ello formulamos la hipótesis de que la percepción de innovación de las marcas del distribuidor generará un efecto de recomendación positivo en el consumidor compartiendo la información y sentimientos generados con las personas bajo su influencia (Mahajan et al., 1984).

H3: Las marcas del distribuidor percibidas como innovadoras generarán un efecto positivo en la recomendación de las mismas.

Ilustramos las relaciones planteadas en las hipótesis mediante el modelo descrito en la figura 1 que pone de manifiesto los efectos intangibles que la innovación percibida en la marca del distribuidor ejercen sobre la confianza, la imagen de la enseña y la tendencia a recomendar.

Figura 1. Efectos de las marcas del distribuidor percibidas como innovadoras

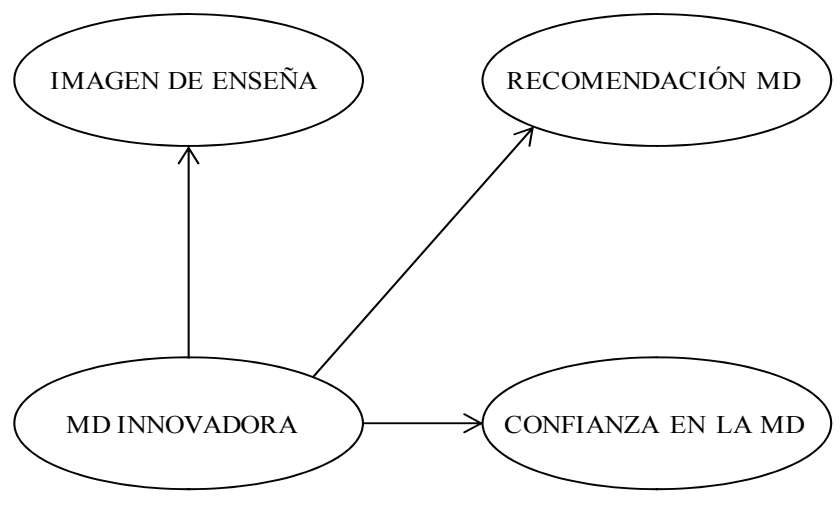




\section{INVESTIGACIÓN EMPÍRICA Y RESULTADOS}

Para la realización de esta investigación se definió como unidad de investigación a los responsables de compra en el hogar que fueron seleccionados por medio de un muestreo aleatorio estratificado. La elección de una muestra estratificada se justifica en la necesidad de obtener información significativa de las principales cadenas detallistas. Por ello se clasificó a los consumidores en estratos según su establecimiento habitual de compra.

Se efectuaron 400 entrevistas personales de las cuales resultaron válidas 384 . Las entrevistas tuvieron lugar a la salida de distintos establecimientos detallistas pertenecientes a las seis enseñas dominantes en España: Carrefour, Alcampo, Lidl, Dia, Mercadona y Eroski. El trabajo de campo se realizó durante una semana, de lunes a sábado durante el mes de mayo de 2012.

Los entrevistados respondieron a preguntas evaluadas con escalas tipo Likert de 7 posiciones donde 1 indicaba el valor máxima disconformidad y 7 el valor de máxima conformidad. El contenido de las escalas estaba relacionado con diferentes áreas de información sobre el comportamiento de compra del consumidor y sus percepciones sobre las marcas del distribuidor y sus enseñas. Todas las variables se midieron utilizando escalas ya contrastadas en la literatura académica.

Para cada individuo se recogió además información sobre el establecimiento de compra que más frecuentaba y la imagen percibida de la enseña (IMAGEN_ENS) según la escala de Grewal et al. (1998) que recogemos en la Tabla 1.

Tabla 1. Escala de Imagen de la enseña, Grewal et al. 1998

\begin{tabular}{lc}
\hline Items & Escala \\
\hline Es un sitio agradable para comprar & $1-7$ \\
Ofrece una experiencia de compra agradable & $1-7$ \\
Tiene una buena imagen de establecimiento & $1-7$ \\
Tiene buen servicio en general & $1-7$ \\
Tiene productos de alta calidad & $1-7$ \\
Tiene vendedores amables & $1-7$ \\
Tiene vendedores expertos & $1-7$ \\
\hline
\end{tabular}


Posteriormente se recogió información sobre si el individuo era comprador de marca del distribuidor o no. En caso afirmativo se midió la percepción que tenía sobre el nivel de innovación de las marcas del distribuidor que compraba (MD_INNOV).

El estudio se centró en las marcas de distribuidor llamadas de tipo tres según la definición de Laaksonen y Reynolds (1994); ya que son el tipo de marca de distribuidor mayoritario tanto en España como en el resto del mundo con la excepción de Estados Unidos y Reino Unido (Anselmsson y Johansson 2007, Geykens et al., 2010).

La confianza que los consumidores encuestados tenían en las marcas del distribuidor que compraban (CONFIA_MD) se midió con la escala de Chaudhuri y Holbrook (2001) que se presenta en la Tabla 2.

Tabla 2. Escala de Chaudhuri y Holbrook (2001) de confianza en la marca

\begin{tabular}{lc}
\hline Items & Escala \\
\hline Confío en esta marca & $1-7$ \\
Me fio de esta marca & $1-7$ \\
Es una marca honesta & $1-7$ \\
Es una marca segura & $1-7$ \\
\hline
\end{tabular}

La posible prescripción o actividad de recomendación boca-oído positiva (RECOM_MD) llevada a cabo por el individuo sobre las marcas del distribuidor se midió a través de la escala de Price y Arnould (1999) que figura en la Tabla 3. 
Tabla 3. Escala de Price Arnould (1999) sobre recomendación positiva

Items Escala

Recomendaría esta marca a quien me pidiese consejo

Por último, la medición de la innovación percibida en la marca del distribuidor (MD_INNOV) fue adaptada de escalas previas (Moreau et al., 2001; Olshavsky y Spreng, 1996). Los ítems de esta escala se detallan en la tabla 4.

Tabla 4. Escala percepción de innovación de la marca del distribuidor.

Para la obtención de los resultados se procedió en primer lugar a verificar la fiabilidad de las escalas usando el indicador alfa de Cronbach. Todos los valores resultaron superiores a 0,7 (Nunnally y Bernstein, 1999) como se observa en la tabla 5 . 
Tabla 5. Indicador de fiabilidad de las escala.

\begin{tabular}{lc}
\hline Escala & alfa de Cronbach \\
\hline Imagen de la enseña (IMAGEN_ENS) & 0,912 \\
Confianza en la marca de distribuidor (CONFIA_MD) & 0,914 \\
Recomendación positiva de la marca de distribuidor (RECOM_MD) & 0,884 \\
Innovación percibida en la marca del distribuidor (MD_INNOV) & 0,889 \\
\hline
\end{tabular}

Atendiendo a los objetivos fijados en la investigación comenzamos por analizar la percepción que tenían los consumidores sobre la innovación de las marcas del distribuidor. Para ello se calcularon los valores descriptivos relativos a los ítems de la escala de innovación contrastando que las medias obtenidas eran significativamente distintas de la posición 4 que reflejaría la indiferencia en la escala usada. Para todos los ítems la diferencia era significativa indicando una percepción superior $(\mathrm{p}<.05)$. Ver tabla 6.

Tabla 6. Descriptivos de la escala de percepción de innovación de la marca del distribuidor

\begin{tabular}{lcccc}
\hline Es una marca del distribuidor... & $\begin{array}{c}\text { Media } \\
\text { Total }\end{array}$ & Moda & $\begin{array}{c}\text { Desviación } \\
\text { típica }\end{array}$ & Sig. \\
\hline con productos que no encuentro en otras marcas & 4,63 & 6 & 1,601 &, 000 \\
con productos innovadores & 4,38 & 5 & 1,632 &, 000 \\
que siempre me sorprende por sus novedades & 4,20 & 5 & 1,616 &, 014 \\
que lanza productos originales & 4,22 & 4 & 1,777 &, 014 \\
$\quad$ con calidad comparable a otras marcas líderes & 5,43 & 4 & 1,260 &, 000 \\
\end{tabular}

A continuación, se comprobó la validez convergente del modelo de medida. Las cargas factoriales de los ítems en sus respectivas variables latentes tomaban valores entre 0,92 y 0,94 indicando un elevado grado de validez convergente (Chin, 1998). 
También se procedió a calcular la validez discriminante de las variables latentes comprobando que la varianza compartida entre pares de constructos es menor a la varianza extraída media para cada constructo individual (Fornell y Larcker, 1981). Todos los indicadores ofrecen valores por encima de los límites recomendados.

A continuación se estimaron las relaciones propuestas en el modelo por medio de la herramienta estadística path analysis PLS (Partial Least Squares). La elección de esta técnica se basó en las peculiaridades propias de esta investigación: (i) el tamaño muestral utilizado se adapta perfectamente a esta metodología, (ii) puede manejar datos en los que se incumple la condición de normalidad, y (iii) tiene un carácter más exploratorio, en situaciones en las que modelos previos no han sido contrastados empíricamente. El software utilizado fue SmartPLS 2.0 (M3) Beta, Hamburg (Ringle et al., 2005).

La tabla 7 presenta la estimación del modelo estructural y el valor de R2 para cada uno de los constructos (Chin, 1998).

Los valores de las R2 calculadas están por encima del límite de 0,1 y todos los coeficientes beta son positivos y estadísticamente significativos.

La estabilidad de las estimaciones de los parámetros se examina mediante el empleo del Boostrap que ofrece el cálculo del error estándar de los parámetros y los valores de la t de Student (Chin, 1998).

Tabla 7. Validez convergente, Validez Discriminante y R cuadrado

\begin{tabular}{cccc}
\hline & Varianza Media & Validez Convergente & $\mathbf{R}^{2}$ \\
\hline MB_INNOVA & 0,699940 & 0,920596 & - \\
IMAGEN_ENSEÑA & 0,667065 & 0,933238 & 0,369487 \\
CONFIA_MB & 0,797261 & 0,940159 & 0,217922 \\
RECOM_MB & 0,819792 & 0,931659 & 0,516868 \\
\hline
\end{tabular}

La tabla 8 presenta los valores de los coeficientes Path $(\mathrm{p}<0,05)$. De acuerdo con los resultados obtenidos podemos concluir que las tres hipótesis planteadas se confirman y son aceptadas.

Tabla 8. Coeficientes y Estadístico $t$ de las relaciones propuestas.

\begin{tabular}{lcccc}
\hline & Coeficientes & $\begin{array}{c}\text { Error } \\
\text { Estándar }\end{array}$ & Estadístico t & $\boldsymbol{p}$ \\
\hline H1: MB_INNOVA $\rightarrow$ IMAGEN_ENSEÑA & 0,40 & 0,09 & 4,28 & 0,000 \\
H2: MB_INNOVA $\rightarrow$ CONFIA_MB & 0,47 & 0,07 & 6,71 & 0,000 \\
H3: MB_INNOVA $\rightarrow$ RECOM_MB & 0,21 & 0,09 & 2,32 & 0,020 \\
\hline
\end{tabular}




\section{CONCLUSIONES E IMPLICACIONES PARA LA GESTIÓN}

El objetivo de este estudio era investigar la percepción que tienen los consumidores españoles sobre la innovación de las marcas del distribuidor y la influencia de ésta en la creación de valores intangibles relacionados con la percepción de las marcas, las enseñas que las soportan y la prescripción de los consumidores.

Los resultados obtenidos muestran que el consumidor español percibe una actividad innovadora en las marcas del distribuidor y que esta percepción de la innovación genera unos efectos positivos en diversas áreas.

En primer lugar se ha contrastado que la innovación de las marcas del distribuidor no sólo produce efectos positivos en cuanto a una diferenciación de la oferta en el mercado sino en la relación de confianza con la marca y su reputación, lo que significa una ventaja competitiva muy relevante respecto al resto de marcas percibidas como no innovadoras.

En segundo lugar, la innovación percibida genera para las marcas del distribuidor una oferta diferenciadora en el mercado y fortalece la relación con sus consumidores a través de una actitud proactiva de recomendación, de la que se benefician tanto la marca del distribuidor como su enseña.

Por tanto, los resultados obtenidos indican que las marcas del distribuidor percibidas como innovadoras afectan positivamente a la imagen de su enseña y a la confianza que el consumidor declara hacia las mismas, generando en el consumidor una prescripción voluntaria y recomendaciones positivas a sus conocidos. Esto es especialmente importante para el fomento de la prueba, adopción y difusión de los productos nuevos en el mercado.

Estos resultados son importantes desde el punto de vista académico por la reducida y casi inexistente literatura sobre los efectos intangibles de la innovación en las marcas del distribuidor. Además para las empresas adquieren importancia por diversas razones que exponemos a continuación.

En primer lugar el consumidor español percibe que las marcas del distribuidor innovan y esto debería hacer reflexionar a algunas opiniones que argumentan que las marcas del distribuidor solo copian. "Perception is reality", la percepción es la realidad y por tanto se debería afrontar la situación de mercado desde esta perspectiva para comprender mejor las razones de compra y el comportamiento en el mercado de los consumidores españoles.

En segundo lugar, la generación de confianza y recomendación positiva son antecedentes de la lealtad actitudinal. Por lo que esta estrategia de innovación de las marcas del distribuidor podría estar contribuyendo no solo a mejorar sus resultados en el mercado sino a estrechar vínculos emocionales con sus consumidores.

Este hecho es especialmente relevante en un contexto donde las marcas de fabricante están reduciendo su actividad innovadora como se ha indicado 
anteriormente. Este impacto positivo en la actitud de los consumidores hacia las marcas del distribuidor puede generar una ventaja competitiva incluso mayor que la generada en la actualidad por el diferencial de precio existente, abocando en el futuro a las marcas de fabricante a una pérdida de cuota de mercado aún mayor que la experimentada en los últimos años.

En tercer lugar, es interesante resaltar la recomendación positiva que produce la innovación percibida en las marcas del distribuidor ya que la recomendación es uno de los vehículos de comunicación más eficaces para generar prueba de nuevos productos y para reducir el riesgo percibido en la compra. Esto es un punto fundamental para las marcas de distribuidor ya que el riego percibido es un obstáculo que los consumidores deben superar para comprar la marca del distribuidor. Por tanto, es de esperar que estas recomendaciones positivas entre consumidores fomenten la prueba y penetración de las marcas del distribuidor en categorías de producto menos desarrolladas, lo que en última instancia fomentaría el crecimiento inter-categorías.

Por último, esta investigación sugiere una reflexión sobre el futuro de las marcas del distribuidor y las marcas de fabricante.

Durante la crisis económica muchos consumidores se están inclinando a comprar las marcas del distribuidor. La pregunta que se plantean diferentes agentes del mercado es qué pasará después. Los estudios realizados al respecto como el de Lamey et al. (2012) apuntan a que tras la crisis, las marcas del distribuidor experimentarán una bajada, pero su cuota se estabilizará en niveles superiores a los que tenían al comienzo de la crisis. Sin embargo, los resultados de esta investigación conducen hacia un pronóstico menos alentador para las marcas del fabricante. Si en esta época de crisis las marcas de fabricante siguen reduciendo sus inversiones y actividad innovadora, mientras las marcas del distribuidor continúan innovando, las marcas del distribuidor saldrán muy reforzadas, tanto en su cuota de mercado como en valor de marca. Las repercusiones de este se harán patentes en el futuro equilibrio entre marcas de fabricante y marcas de distribuidor en el mercado.

\section{BIBLIOGRAFÍA}

AAKER, D.A. (1991) Managing Brand Equity: Capitalizing on the Value of a Brand Name. The Free Press, New York, 1991.

AAKER, D.A. (1996). Measuring brand equity across products y markets. California Management Review, Vol. 38, no 3.

AAKER, D.A. (2001) Building strong brands. The Free Press, New York.

ABRIL, C., GAVILAN, D. y AVELLO, M. (2009) Influence of the perception of different types of store brands on consumer typologies and satisfaction levels. Innovative Marketing, Vol. 5, no 4. p.75-82 
ABRIL, C., GAVILAN, D. y AVELlO, M (2010) Positioning of Spanish Retailers. Perception of high frequency versus low frequency consumers. AdReserach, julio-diciembre. p.26-39

AILAWADI, K.L. y Keller, K. (2004) Understanding retail branding: Conceptual insights y research priorities. Journal of Retailing, Vol. 80, no. 4. p. 331-342.

ANSELMSSON, J., JOHANSSON, U. y PERSSON, N. (2004) Understanding price premium for grocery products: a conceptual model of customer-based brand equity. Journal of Product y Brand Management, Vol.16, no 6. p. 401414.

BERRY, LEONARD L. (2000) Cultivating service brand equity, Journal of the Academy of Marketing Science, Vol. 28. no1. p. 128-137.

CHAUDHURI, A. y HOLBROOK, M. (2001) The chain effects from brand trust $y$ brand affect to brand performance: The role of brand loyalty. Journal of Marketing, Vol. 65. p. 81-93.

CHIN, W. (1998) Issues y Opinions on Structural Equation Modeling. MIS Quarterly, Vol. 22, no 1.p. 7-16.

COLLINS-DODD, C. y LINDLEY, T. (2003) Store brands y retail differentiation: the influence of store image y store brand attitude on store own brand perceptions. Journal of Retailing y Consumer Services, Vol. 10. p. 345-352.

DATAMONITOR (2012) Datamonitor's Product Launch Analytics Packaged Facts Report.

DELVECCHIO, D. (2001) Consumer perceptions of private label quality: the role of product category characteristics y consumer use of heuristics, Journal of Retailing y Consumer Services, Vol. 8. p. 239-249.

FORNELL, C. y LARCKER, D.F. (1981) Evaluating Structural Equations Models with Unobservable Variables y Measurement Error. Journal of Marketing Research, Vol. 18. p. 39-50.

GARRETSON, J.A., FISHER, D. y BURTON, S. (2002) Antecedents of private label attitude y national brand promotion attitude: Similarities y differences, Journal of Retailing, Vol. 78, no 2. p. 91- 101.

GEYSKENS, I., GIELENS, K. y GIJSBRECHTS, E. (2010) Proliferating privatelabel portfolios: how introducing economy y premium private labels influences brand choice. Journal of Marketing Research, Vol 4, no 5. p. 791-807.

GREWAL, D.R., KRISHNAN, J.B. y NORM, B. (1998) The effect of store name, brand name y price discounts on consumers' evaluations y purchase intentions. Journal of Retailing, Vol. 74, no 3. p. 331-352.

JOHNSON, MICHAEL D. y FRED SELNES. (2004) Customer Portfolio Management: Toward a Dynamic Theory of Exchange Relationships. Journal of Marketing, Vol. 68, no 2. p. 1-17.

KUMAR, N. y STEENKAMP, J.B. (2007) Private label strategy: How to meet the store brand challenge. Harvard Business School Press, Boston, MA. 
LAAKSONEN, H., REYNOLDS, J. (1994) Opportunities for own brands in European grocery retailing. European Retail Digest, Vol. 94, no 3. p. 4-8.

LAMEY, L., DELEERSNYDER, B., STEENKAMP, J.B. y DEKIMPE, M. (2012) The Effect of Business-Cycle Fluctuations on Private-Label Share: What Has Marketing Conduct Got to Do with It? Journal of Marketing, Vol. 76, no 1. p. 1-19.

MAHAJAN, V., MULLER, E. y KERIN, R. (1984). Introduction Strategy for New Products with Positive y Negative Word-of-Mouth, Management Science, Vol. 30, no 12. p. 1389-1404.

MORGAN, R.M. y HUNT, S.D. (1994) The commitment-trust theory of relationship marketing. Journal of Marketing, Vol. 58, no 3. p. 20-38.

MOREAU, C., LEHMANN, D. y MARKMAN, A. (2001) Entrenched Knowledge Structures y Consumer Response to New Products, Journal of Marketing Research. Vol. 38, no 1.p. 14-29.

MINTEL (2009) http://www.foodnavigator-usa.com/Business/Private-labelgrowth-driven-by-innovation-says-Mintel. Último acceso 8 abril 2013.

MOLINA-CASTILLO, J.F. y MUNUERA-ALEMÁN, J.L. (2008) Efectos de la novedad y de la calidad del producto en el resultado a corto y a largo plazo en las empresas innovadoras españolas, Universia Business Review, Vol. 20. p. 68-83.

NUNNALLY J.C. y BERNSTEIN I.H. Psychometric theory. 3rd ed. New York: Mc Graw-Hill;1999.

OLSHAVSKY, R. y SPRENG, R. (1996) An exploratory study of the innovation evaluation process. Journal of product innovation management, Vol. 13, no 6. p. 512-529.

PAUWELS, K. y SRINIVASAN, S. (2004) Who benefits from store brand entry? Marketing Science, Vol. 23, no 3. p. 364-390.

PRICE, L.L. y ARNOULD, E.J. (1999) Commercial Friendships: Service Provider--Client Relationships in Context. Journal of Marketing, Vol. 63, no 4. p. 38-56.

RINGLE, C.M., WENDE, S. Y WILL, S. (2005) SmartPLS 2.0 (M3) Beta, Hamburg.

SPROTT, D.E. y SHIMP, T.A. (2004) Using product sampling to augment the perceived quality of store brands. Journal of Retailing, Vol. 80, no 4. p. 305315.

SRIRAM, S., SUBRAMANIAN B., MANOHAR U.K. (2007) Monitoring the dynamics of brand equity using store-level data. Journal of Marketing. Vol. 71. p. 61-78.

SYMPHONY IRI. (2012) La marca de distribución en España, un avance cualitativo. Marketing News. 25 enero. 
TENENHAUS, M., ESPOSITO, V., CHATELIN, Y.M. y LAURO, C. (2005) PLS Path Modeling. Computational Statistics y Data Analysis, Vol. 48, no 1. p. 159 $-205$.

VERHOEF, P.C., NIJSSEN, E.J. y SLOOT, L.M. (2002) Strategic reactions of national brand manufacturers towards private labels. European Journal of Marketing, Vol. 36, no 11. p. 1309-1326.

WOLD, H. (1985) Partial least squares. Encyclopedia of Statistical Sciences, Vol. 6. Wiley, New York, p. 581-591. 